XXXIX "Jaszowiec" International School and Conference on the Physics of Semiconductors, Krynica-Zdrój 2010

\title{
Raman Studies of Defects in Graphene Grown on SiC
}

\author{
K. Grodecki ${ }^{a, b}$, R. BożeK ${ }^{b}$, J. Borysiuk $^{b, c}$, W. Strupinski ${ }^{a}$, A. Wysmolek $^{b}$, \\ R. STĘPNIEWSKI ${ }^{b}$ AND J.M. BARANOWSKI ${ }^{a, b}$ \\ ${ }^{a}$ Institute of Electronic Material Technology, Wólczyńska 133, 01-919 Warsaw, Poland \\ ${ }^{b}$ Institute of Experimental Physics, Faculty of Physics, University of Warsaw, Hoża 69, 00-681 Warsaw, Poland \\ ${ }^{c}$ Institute of Physics, Polish Academy of Sciences, al. Lotników 32/46, 02-668 Warsaw, Poland \\ The Raman scattering studies of multi-layer graphene obtained by high temperature annealing of carbon \\ terminated face of $4 \mathrm{H}-\mathrm{SiC}(000-1)$ substrates are presented. Intensity ratio of the $D$ and $G$ bands was used to \\ estimate the average size of the graphene flakes constituting carbon structures. The obtained estimates were \\ compared with flake sizes from atomic force microscopy data. We found that even the smallest structures observed \\ by atomic force microscopy images are much bigger than the estimates obtained from the Raman scattering \\ data. The obtained results are discussed in terms of different average flake sizes inside and on the surface of the \\ multi-layer graphene structure, as well as different type of defects which would be present in the investigated \\ structures apart from edge defects.
}

PACS: $61.48 . \mathrm{Gh}, 78.30 . \mathrm{Fs}$

\section{Introduction}

In recent years much attention has been devoted to graphene as a potential successor of silicon in electronic applications. Thermal decomposition of silicon carbide ( $\mathrm{SiC}$ ) substrates seems to be one of promising methods of growth of large homogeneous graphene structures. It was shown that this method could be transferred to standard chemical vapor deposition (CVD) reactors used for $\mathrm{SiC}$ epitaxial growth [1]. This allows to control the growth rate of the graphene layers by changing argon pressure in the reactor, and opens new possibilities in graphene growth control making it more compatible with nowadays SiC technology.

The Raman scattering has been proven as a powerful characterization method of carbon-based structures [2]. The Raman spectra of graphene-like structures consist of several bands. The most interesting are the so-called $D$, $G$ and $2 D$ bands $[2,3]$. From the analysis of the Raman spectra several parameters can be estimated, including the actual number of graphene layers in the sample, a type of stacking and strain state $[3,4]$.

It is generally accepted that by measuring intensity ratio of the $D$ and $G$ bands the average size of the graphene flakes constituting carbon structures can be calculated [5]. This idea is based on the fact that the $D$ -band intensity is induced by the presence of defects. In the case of perfect graphene flakes such defects are localized on the edges, thus $D$-band intensity is proportional to the length of edges of the graphene flakes. Assuming that the $G$-band intensity is proportional to the flake area, the $D$ to $G$ intensity ratio should be directly related to the average flake size [5].

In this study we verify to what extent the above assumption is fulfilled. The ratio of $G$ - and $D$-band intensities is examined for several multilayer graphene structures grown on $4 \mathrm{H}-\mathrm{SiC}(000-1)$ on-axis substrates.

\section{Experimental details}

The investigated samples were fabricated in hot-wall CVD reactor Epigress VP508. Prior to the annealing in situ etching process of the $\mathrm{SiC}$ substrates was carried out in hydrogen and propane atmosphere. After etching on some substrates epitaxial $\mathrm{SiC}$ layers were deposited. Then annealing at temperature of $1600^{\circ} \mathrm{C}$ was carried out. Different annealing time and argon pressure in the reactor were used. The obtained samples were characterized using the Raman spectroscopy and atomic force microscopy (AFM). Micro-Raman scattering experiments were performed at room temperature, in backscattering geometry, using $532 \mathrm{~nm}$ line from Nd-YAG as a source of continuous wave excitation. Laser spot size on the sample surface was of about $2 \mu \mathrm{m}$. For each spectrum the $\mathrm{SiC}$ phonon Raman signal was subtracted. The actual number of graphene layers deposited was verified by optical absorption [6].

\section{Results and discussion}

Representative Raman spectra obtained for selected graphene structures are presented in Fig. 1. From the analysis of the intensity ratio between $G$ - and $D$-bands we determined the average size of graphene flakes of $40 \mathrm{~nm}$ for the 8 monolayer sample, $500 \mathrm{~nm}$ for the 10 monolayer sample and $100 \mathrm{~nm}$ for the sample with 14 monolayer sample grown at 100 mbar, 50 mbar, and 100 mbar argon pressure, respectively. For the sample 14 monolayer sample epitaxial $4 \mathrm{H}-\mathrm{SiC}$ substrate, grown on the $\mathrm{SiC}$ substrate prior to the annealing, was used. AFM images of the same graphene structures are presented in Fig. 2.

As a measure of graphene flakes size, an average size of smallest regions defined by white narrow lines on the AFM image, corresponding to the so-called puckers [7], was used. For the 8, 10, and 14 monolayer samples the size of $1,1.5$ and $1.5 \mu \mathrm{m}$ was obtained, respectively. 


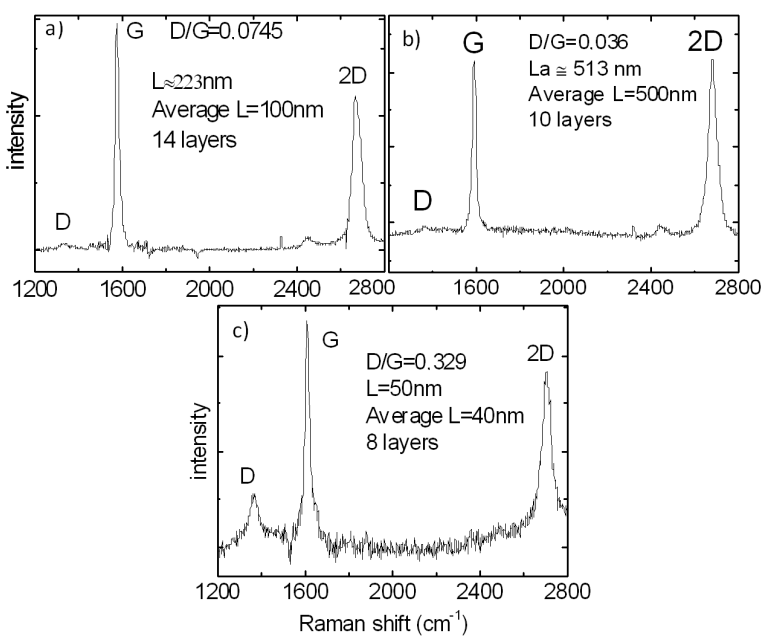

Fig. 1. Representative Raman spectra of multilayer graphene samples consisting of (a) 14 layers, (b) 10 layers, (c) 8 layers.



Height $55 \mathrm{~nm}$



Fig. 2. AFM images of selected positions on the multilayer graphene samples composed of (a) 14 layers, (b) 10 layers, (c) 8 layers.

The comparison of two samples grown at the same pressure suggest that quality of the used $\mathrm{SiC}$ has significant influence on the size of graphene flakes. Differences in average size of the flakes for the samples grown at 50 mbar and 100 mbar on the same substrate suggest that size of the graphene flakes decreases with the increasing pressure in the reactor.

\section{Conclusions}

We have estimated the size of the graphene flakes in multi-layer graphene structures grown on $\mathrm{SiC}$ substrate using two methods: direct AFM observation of the sample surface and the analysis of the Raman scattering measurements $(G / D$-band intensity ratio). Comparison of size flakes for samples annealed at different conditions or different substrates (bulk $\mathrm{SiC}$ or epitaxial layer) suggests that quality of substrate and the pressure in the reactor has a significant influence on the actual flake dimensions. We found that even the smallest structures deduced from AFM images have much bigger diameter than it can be estimated from the Raman data analysis. It could suggest that our AFM measurements are not enough precise to reveal such small structures. This could mean that the sizes of the graphene flakes are not equal in all layers constituting a particular sample. It may suggest that layers beneath the top layer are formed from smaller flakes. This is hidden to AFM measurements, but can manifest itself in the Raman spectra. Thus one could expect that graphene flakes formed close to the interface are rather small, and grow in size being already separated from the interface. This idea needs to be verified by further experiments.

\section{Acknowledgments}

The work was partially supported by Polish Ministry of Science and Higher Education, projects No. 670/N- and No. 671/N-ESF-EPI/2010/0 within the EuroGRAPHENE program of the European Science Foundation, and grant No. 395/N-PICS-FR/2009/0.

\section{References}

[1] W. Strupiński, A. Drabińska, R. Bożek, J. Borysiuk, A. Wysmolek, R. Stepniewski, K. Kościewicz, P. Caban, K. Korona, K. Grodecki, P.A. Geslin, J.M. Baranowski, Mater. Sci. Forum 645-648, 569 (2010).

[2] C. Faugeras, A. Nerrière, M. Potemski, A. Mahmood, E. Dujardin, C. Berger, W.A. de Heer, Appl. Phys. Lett. 92, 011914 (2008).

[3] N. Camara, J.-R. Huntzinger, G. Rius, A. Tiberj, N. Mestres, F. Pérez-Murano, Ph. Godignon, J. Camassel, Phys. Rev. B 80, 125410 (2009).

[4] T. Yu, Z. Ni, C. Du, Y. You, Y. Wang, Z. Shen, Phys. Chem. C 112, 12602 (2008).

[5] M.A. Pimenta, G. Dresselhaus, M.S. Dresselhaus, L.G. Cançado, A. Jorio, R. Saito, Phys. Chem. Chem. Phys. 9, 1276 (2007).

[6] A. Drabińska, K. Grodecki, W. Strupiński, R. Bożek, K.P. Korona, A. Wysmołek, R. Stępniewski, J.M. Baranowski, Phys. Rev. B 81, 245410 (2010).

[7] J. Hass, W.A. de Heer, E.H. Conrad, J. Phys., Condens. Matter 20, 323202 (2008). 